University of Nebraska - Lincoln

DigitalCommons@University of Nebraska - Lincoln

2010

Surgical intervention for infective endocarditis in a veteran population

Lorraine D. Cornwell

Baylor College of Medicine

Danny Chu

Baylor College of Medicine

Scott A. LeMaire

Baylor College of Medicine

Joseph Huh

Baylor College of Medicine

Shubhada Sansgiry

Michael E. DeBakey Veterans Affairs Medical Center

See next page for additional authors

Follow this and additional works at: https://digitalcommons.unl.edu/publichealthresources

Part of the Public Health Commons

Cornwell, Lorraine D.; Chu, Danny; LeMaire, Scott A.; Huh, Joseph; Sansgiry, Shubhada; Coselli, Joseph S.; and Bakaeen, Faisal G., "Surgical intervention for infective endocarditis in a veteran population" (2010). Public Health Resources. 166.

https://digitalcommons.unl.edu/publichealthresources/166

This Article is brought to you for free and open access by the Public Health Resources at DigitalCommons@University of Nebraska - Lincoln. It has been accepted for inclusion in Public Health Resources by an authorized administrator of DigitalCommons@University of Nebraska - Lincoln. 


\section{Authors}

Lorraine D. Cornwell, Danny Chu, Scott A. LeMaire, Joseph Huh, Shubhada Sansgiry, Joseph S. Coselli, and Faisal G. Bakaeen 


\title{
Surgical intervention for infective endocarditis in a veteran population
}

\author{
Lorraine D. Cornwell, M.D. ${ }^{a, b}, *$, Danny Chu, M.D..$^{a, b, c}$, Scott A. LeMaire, M.D. ${ }^{a, c}$, \\ Joseph Huh, M.D. ${ }^{a, b, c}$, Shubhada Sansgiry, Ph.D. ${ }^{a, b}$, Joseph S. Coselli, M.D. ${ }^{a, c}$, and \\ Faisal G. Bakaeen, M.D. ${ }^{a, b, c}$
}

\begin{abstract}
${ }^{a}$ Division of Cardiothoracic Surgery, Michael E. DeBakey Department of Surgery, Baylor College of Medicine, Houston, TX, USA; ${ }^{b}$ Michael E. DeBakey Veterans Affairs Medical Center, Houston, TX, USA; ${ }^{c}$ Texas Heart Institute at St Luke's Episcopal Hospital, Houston, TX, USA
\end{abstract}

\section{KEYWORDS: \\ Endocarditis; \\ Cardiac surgery; \\ Mortality; \\ Morbidity; \\ Outcomes; \\ Veterans}

\begin{abstract}
BACKGROUND: Surgical management of infective endocarditis can be challenging. Veteran patients are unique because they often have significant comorbidities, and surgical management of endocarditis in this population has not been well described.

METHODS: Using a prospective database, 46 consecutive patients who underwent valve surgery for acute infective endocarditis between 1987 and 2009 were identified. Survival was assessed using the Kaplan-Meier method.

RESULTS: All patients were men (mean age, $56 \pm 9$ years). The most common indication for surgical intervention was congestive heart failure (60\%). The aortic valve was the only valve infected in most patients (65\%). Operative morbidity and mortality were $33 \%$ and $9 \%$, respectively. The 1 -year, 3 -year, 5-year, and 10-year unadjusted survival rates were $72 \%, 57 \%, 51 \%$, and $30 \%$, respectively.

CONCLUSIONS: Although acceptable short-term outcomes can be achieved in veterans undergoing surgical treatment for endocarditis, unadjusted long-term survival may be poor.

Published by Elsevier Inc.
\end{abstract}

Infective endocarditis is an uncommon but life-threatening disease, with an estimated incidence of 3 to 10 episodes per 100,000 person-years and mortality of up to $50 \%{ }^{1}$ Despite improved antibiotic regimens and critical care, the management of infective endocarditis remains a challenge. Surgical intervention may be lifesaving, but it is difficult to determine which patients are most likely to benefit. Indications for surgery continue to evolve but often include in-

This paper was presented at the annual meeting of the Association of VA Surgeons, Indianapolis, Indiana, May 9 to 11, 2010.

* Corresponding author: Tel.: 212-717-0123; fax: 713-794-7352.

E-mail address: cornwell@bcm.edu

Manuscript received May 12, 2010; revised manuscript July 7, 2010 tractable heart failure, embolic events, the presence of infected prosthetic material, and unresolved sepsis. ${ }^{2}$ Although operative mortality appears to be improving, it remains substantial, down from $30 \%$ in the past 2 decades to a reported $12 \%$ in more recent studies. ${ }^{3,4}$

Veterans are a unique patient population, because they often have significant comorbidities. Surgical management of endocarditis in this population has not been adequately described, and findings from surgical studies of other patient populations may not apply to the veteran population. Because evaluation of surgical outcomes in the veteran population is needed to help direct management and determine prognoses, we report the indications and outcomes of surgical management of endocarditis in patients at a US Department of Veterans Affairs (VA) facility. 
Table 1 Risk profile and preoperative status of veterans with endocarditis $(n=46)$

\begin{tabular}{lc}
\hline Characteristic & Value \\
\hline Age (y) & $56 \pm 9$ \\
Men & $46(100)$ \\
Previous valve surgery & $3(6.5)$ \\
Diabetes & $7(15)$ \\
Cerebrovascular disease & $14(30)$ \\
Active smokers & $17(37)$ \\
Chronic obstructive pulmonary disease & $16(35)$ \\
Pulmonary rales & $38(83)$ \\
New York Heart Association class III & \\
$\quad$ or IV symptoms & $35(76)$ \\
Concomitant coronary artery disease & $5(11)$ \\
Previous myocardial infarction & $11(24)$ \\
Emergency operation & $22(48)$ \\
Valves involved & \\
$\quad$ Aortic (isolated) & $30(65)$ \\
Mitral (isolated) & $8(17)$ \\
Tricuspid (isolated) & $1(2)$ \\
Multiple valves & $7(15)$ \\
\hline
\end{tabular}

Data are expressed as mean \pm SD or as number (percentage).

\section{Methods}

This study was granted waiver of informed consent and approved by the institutional review boards of the Michael E. DeBakey VA Medical Center (MEDVAMC) and Baylor College of Medicine. Data on 46 consecutive patients who underwent valve surgery for active infective endocarditis from January 1987 to January 2009 at the MEDVAMC were analyzed. Data were collected from the MEDVAMC's Continuous Improvement in Cardiac Surgery Program database and supplemented by a thorough review of the patients' medical records. Access to complete medical records was possible for 32 patients.

The Continuous Improvement in Cardiac Surgery Program is a prospective database, organized by the VA, to provide continuous assessment and improved quality of care for all patients who undergo cardiac surgery at VA hospitals and contains comprehensive data on $>140$ variables, including demographic, clinical, outcome, and resource variables. ${ }^{5-7}$ The death index component of the database was obtained from the Beneficiary Identification Records Locator Subsystem death file, which contains information about veterans known to have died from any cause.

During all procedures, cardiopulmonary bypass, systemic hypothermia, and cardioplegia for myocardial protection were used. Intraoperative transesophageal echocardiography was performed in all patients for whom complete records were available. The operative procedure included debridement of all affected areas, copious irrigation, reconstruction of any defects, and either valve repair or replacement with a prosthetic valve. Aortic root replacement with a homograft was preferred in the presence of destructive aortic root infection or abscess.
Data were analyzed using SAS version 9.1 (SAS Institute Inc, Cary, NC) to assess unadjusted survival using KaplanMeier methodology.

\section{Results}

All 46 patients were men, with a mean age of $56 \pm 9$ years. The risk profiles of the patients are summarized in Table 1. Forty-three patients (93\%) had native valve endocarditis, and 3 (7\%) had prosthetic valve endocarditis. The aortic valve was the only valve infected in 30 of the patients $(65 \%)$, and multiple valves were involved in $7(15 \%)$. Aortic root replacement with homografts was performed in 7 patients (15\%) and valve repair in $5(11 \%)$.

Information about clinical factors, such as blood culture results and echocardiographic findings, was obtained from the medical records in 32 of the patients (70\%) (Table 2). Most patients underwent operations early in their courses of illness, the vast majority (81\%) within 1 week from the time of surgical consultation. Although many patients had $>1$ indication for operative intervention, the most common was congestive heart failure (60\% of patients). Evidence of preoperative emboli was present in $25 \%$ of patients. Trans-

Table 2 Clinical characteristics of veterans with endocarditis $(n=32)$

\begin{tabular}{lr}
\hline Characteristic & $\mathrm{n}(\%)$ \\
\hline Organisms by blood culture & \\
Staphylococcus & $11(34)$ \\
Streptococcus & $6(19)$ \\
Enterococcus & $7(22)$ \\
Culture negative & $6(19)$ \\
Other & $2(6)$ \\
Fever & $21(66)$ \\
Elevated white blood cell count & $20(63)$ \\
Operative indications & \\
Congestive heart failure & $19(60)$ \\
Uncontrolled infection & $9(28)$ \\
Cardiogenic or septic shock & $5(16)$ \\
Preoperative embolic event & $8(25)$ \\
Preoperative echocardiographic findings & $12(38)$ \\
Perivalvular abscess & $29(91)$ \\
New valve regurgitation on echocardiography & $18(56)$ \\
Vegetation on preoperative echocardiography & $17(53)$ \\
Time from diagnosis to operation < 1 wk & \\
Time from surgical consultation to operation & $26(81)$ \\
$\quad<1$ wk & $16(50)$ \\
Source of infection & $27(84)$ \\
Intravenous drug abuse & $12(38)$ \\
Soft-tissue infection & $15(47)$ \\
Pyelonephritis & \\
Dental & $2(22)$ \\
Dialysis & $2(6)$ \\
Other/unknown & $2(6)$ \\
Operative findings & $1(3)$ \\
Vegetation & \\
Perivalvular abscess & \\
Valve perforation & \\
\hline & \\
& \\
&
\end{tabular}


Table 3 Operative morbidity and mortality in veterans with endocarditis $(n=46)$

\begin{tabular}{lc}
\hline Operative Result & $\mathrm{n}(\%)$ \\
\hline Operative mortality & $4(9)$ \\
Perioperative complications & \\
Reexploration for bleeding & 0 \\
Myocardial infarction & 0 \\
Stroke & 0 \\
Mediastinitis & $1(2)$ \\
Heart block & $2(4)$ \\
Prolonged ventilation & $10(22)$ \\
Renal failure & $1(2)$ \\
Recurrent endocarditis & $4(9)$ \\
Total patients with complications & $15(33)$ \\
\hline
\end{tabular}

esophageal echocardiography identified valvular abscesses in $38 \%$ of patients and vegetations in $56 \%$ of patients (average size, $9.8 \mathrm{~mm}$ ). Results of cultures of excised tissues were positive in $47 \%$ of patients.

Operative mortality was $9 \%$, and morbidity was $33 \%$. Postoperative complications are summarized in Table 3. Recurrent endocarditis occurred in 4 patients (9\%), all of whom were treated medically. Four patients died, 3 of low cardiac output on the day of surgery and 1 of multiple-organ failure after 30 days in the intensive care unit. All 4 of these patients required emergency aortic valve surgery, and 2 were in preoperative cardiogenic or septic shock. In addition, valvular abscesses necessitating homograft root replacement were present in 2 of these 4 patients.

Unadjusted survival was $72 \%$ at 1 year $(95 \%$ confidence interval $[\mathrm{CI}], 56 \%-83 \%), 57 \%$ at 3 years $(95 \% \mathrm{CI}, 41 \%-$ $70 \%), 51 \%$ at 5 years (95\% CI, $35 \%-65 \%)$, and $30 \%$ at 10 years (95\% CI, 15\%-46\%) (Fig. 1).

\section{Comments}

Our results reflect a tendency in our facility toward early surgical management, with an acceptably low operative morbidity and mortality despite a critically ill patient population. A trend toward early mortality risk was noted in patients with hemodynamic compromise and with advanced infection requiring more extensive surgery. We believe that early referral for surgery, before patients have irreversible end-organ damage from heart failure or sepsis, is important for minimizing operative morbidity and mortality.

Our short-term results compare favorably with those of other recent surgical series. ${ }^{3,4}$ Although age, persistent infection, renal failure, shock, prosthetic endocarditis, and impaired ventricular function have been identified as predictors of poor survival, ${ }^{3,4,8,9}$ our study's small sample size precluded us from confirming the importance of these factors.

Indications for surgery had a similar distribution to those of other surgical series of active complicated endocarditis. ${ }^{3,4,8}$ The most common indication for urgent surgical intervention is usually intractable heart failure, as was the case for $60 \%$ of the patients in our study. Twenty-eight percent of our patients had uncontrolled infection, another indication for early surgery. Several of the patients in our study had multiple indications for surgical intervention, including embolic events, perivalvular abscess, shock, and multidrug-resistant organisms.

Although surgical risk may be higher during the acute phase of endocarditis infection, there are clearly times when earlier surgical intervention is lifesaving. In fact, early surgery is now thought to be an important determinant of outcomes in complicated endocarditis, with studies showing a clinically and statistically significant difference in mortal-

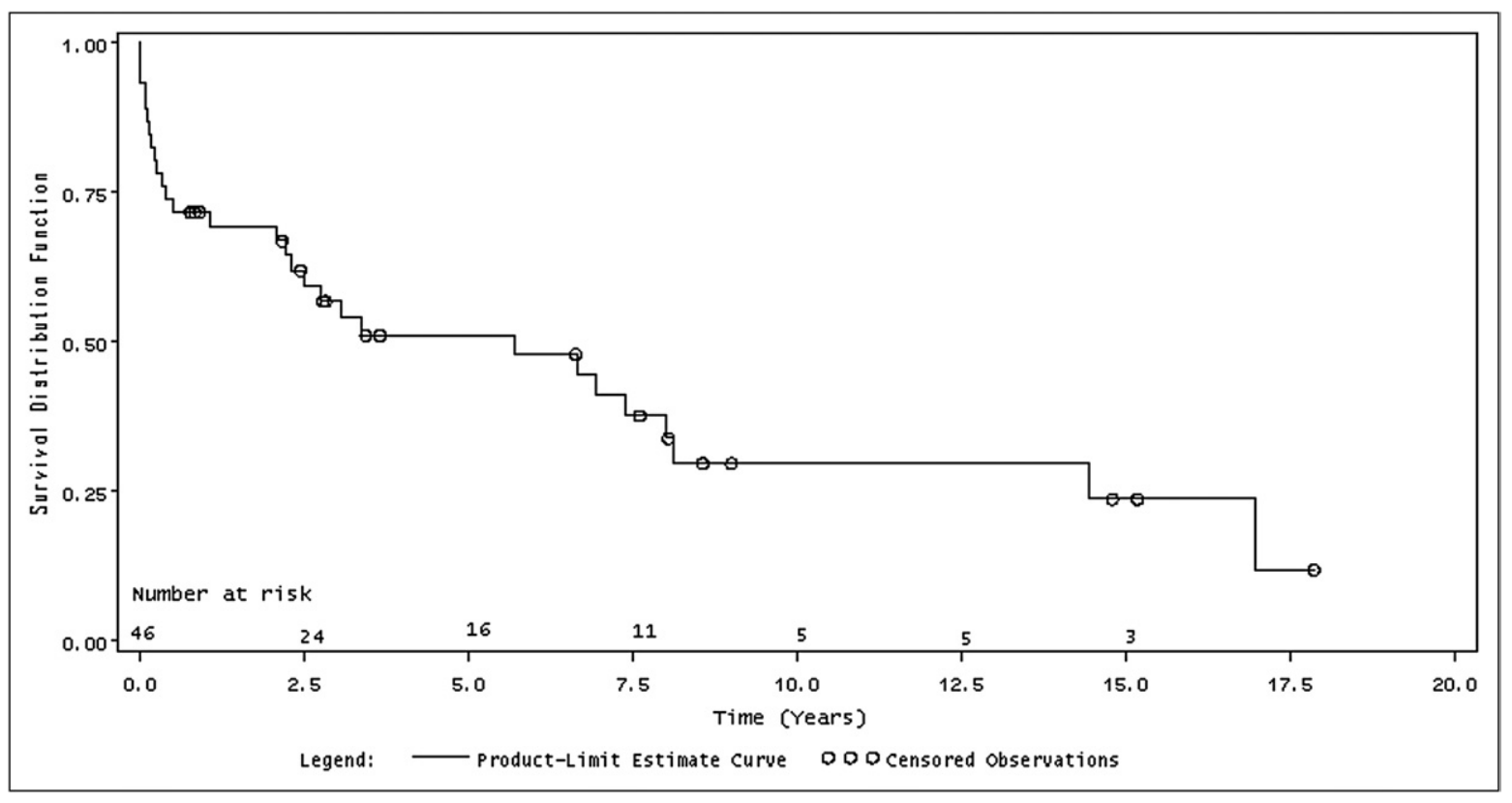

Figure 1 Kaplan-Meier survival curve in veterans with endocarditis. 
ity between patients treated medically (32\%) and surgically $(<20 \%){ }^{10-12}$

More controversial is the exact role of surgery in preventing embolic events in uncomplicated endocarditis. In our study, only 1 patient underwent surgery for the sole indication of a large, mobile vegetation. Although embolic events are common in infective endocarditis (a reported $20 \%-50 \%$ ), they are most often a presenting symptom and usually occur before antibiotic therapy is initiated. After antibiotics are started, the risk for new embolic events is $3 \%$ to $21 \%$ and mostly within the first week of illness. ${ }^{1,2,13}$ Theoretically, surgeons could focus on early operation only for those patients with increased risk for emboli, which includes Staphylococcus infection, mobile vegetations, mitral valve vegetations, and vegetations $>1 \mathrm{~cm}$ (highest risk if vegetations are $>1.5 \mathrm{~cm}){ }^{1,2,13,14}$ However, results from one study refute the notion that vegetation size is associated with increased embolic risk, ${ }^{15}$ and surgical risk may be higher in the acute phase of illness. Thus, the value of early surgery for vegetations alone is still questioned.

Periannular extension of infection or abscess, which occurred in $38 \%$ of our patients, is common in aortic valve endocarditis $(10 \%-40 \%)^{16}$ and is predictive of higher mortality. ${ }^{3,17}$ Two of the 4 early deaths in our study involved patients with extensive periannular aortic destruction reconstructed with homograft root replacement. The use of homografts in the presence of severe tissue destruction has been shown to help considerably with reconstruction of the aortic root, ${ }^{18}$ with a low incidence of sewing-ring infections. ${ }^{19,20}$ However, Avierinos et $\mathrm{al}^{21}$ found that conventional prostheses implanted during the active phase of aortic endocarditis have a similar long-term prognosis as that of homografts, so the increased surgical time required to place a homograft may not be worth the risk if there is not significant root destruction. ${ }^{21}$

The unadjusted long-term survival for patients in our study was poor compared with that reported in other studies, with 10 -year survival of $30 \%$ in our study versus $52 \%$ to $73 \%$ in other studies. ${ }^{3,4,8}$ The difference may be due in part to the relatively older age of our patients, who had a mean age of $56 \pm 9$ years compared with a mean patient age of about 50 years in other studies, and to the strength of the database we used in reporting late deaths. However, these results may also indicate that veterans with serious illnesses such as endocarditis have an increased long-term mortality risk, which may be worth taking into consideration when advising patients. For example, on the basis of this study, decisions concerning use of bioprosthetic valves in veterans with active endocarditis should not be dictated by age alone. Valve deterioration is less likely to become an issue, because longevity appears to be decreased in this population.

Strengths of our study include its long-term and complete follow-up, a unique patient cohort, and a strong and complete database. Also, ours is one of the few reports on the surgical management and outcomes of active endocarditis in veterans; the only other report we could find with regard to endocarditis in this patient population addressed only prosthetic valve endocarditis in a multicenter study. ${ }^{22}$ Finally, the reporting of early and late outcomes can be an important determinant for risk assessment and surgical decision making.

Limitations of this study include those related to any retrospective analyses and to its relatively small sample size. Complete medical records were not available for all patients, which limited the available information on pathogenic and echocardiographic findings. In addition, because of the long study period, several different surgeons were involved, and surgical techniques and antibiotic therapy have evolved. However, indications for surgery remain essentially unchanged, and the need for early surgical referral for complicated endocarditis is reinforced by our findings of low operative morbidity and mortality, which compare favorably with the results of other studies of medically managed patients with complicated endocarditis. ${ }^{11}$

Surgical treatment of veterans with active endocarditis can be accomplished safely, with low operative mortality and morbidity. Early surgical referral is advisable. On the basis of our data, relatively poor unadjusted long-term survival should be anticipated in the veteran population.

\section{Acknowledgments}

Chrissie Chambers, M.A., E.L.S., contributed to the editing of the manuscript, and Stephen N. Palmer, Ph.D., E.L.S., of the Texas Heart Institute reviewed the proofs.

\section{References}

1. Moreillon P, Que YA. Infective endocarditis. Lancet 2004;363: 139-49.

2. Baddour LM, Wilson WR, Bayer AS, et al. Infective endocarditis: diagnosis, antimicrobial therapy, and management of complications. Circulation 2005;111:e394-434.

3. David TE, Gavra G, Feindel CM, et al. Surgical treatment of active infective endocarditis: a continued challenge. J Thorac Cardiovasc Surg 2007;133:144-9.

4. Kaiser SP, Melby SJ, Zierer A, et al. Long-term outcomes in valve replacement surgery for infective endocarditis. Ann Thorac Surg 2007; 83:30-5.

5. Grover FL, Hammermeister KE, Burchfiel C. Initial report of the Veterans Administration Preoperative Risk Assessment Study for Cardiac Surgery. Ann Thorac Surg 1990;50:12-26.

6. Grover FL, Johnson RR, Shroyer AL, et al. The Veterans Affairs Continuous Improvement in Cardiac Surgery Study. Ann Thorac Surg 1994;58:1845-51.

7. Grover FL, Shroyer AL, Hammermeister KE. Calculating risk and outcome: the Veterans Affairs database. Ann Thorac Surg 1996;62: S6-11.

8. Alexiou C, Langley SM, Stafford H, et al. Surgery for active culturepositive endocarditis: determinants of early and late outcome. Ann Thorac Surg 2000;69:1448-54.

9. Jassal DS, Neilan TG, Pradhan AD, et al. Surgical management of infective endocarditis: early predictors of short-term morbidity and mortality. Ann Thorac Surg 2006;82:524-9. 
10. Bishara J, Leibovici L, Gartman-Israel D, et al. Long-term outcome of infective endocarditis: the impact of early surgical intervention. Clin Infect Dis 2001;33:1636-43.

11. Hasbun R, Vikram HR, Barakat LA, et al. Complicated left-sided native valve endocarditis in adults: risk classification for mortality. JAMA 2003;289:1933-40.

12. Netzer RO, Altwegg SC, Zollinger E, et al. Infective endocarditis: determinants of long term outcome. Heart 2002;88:61-6.

13. Dickerman SA, Abrutyn E, Barsic B, et al. The relationship between the initiation of antimicrobial therapy and the incidence of stroke in infective endocarditis: an analysis from the ICE Prospective Cohort Study (ICE-PCS). Am Heart J 2007;154:1086-94.

14. Tischler MD, Vaitkus PT. The ability of vegetation size on echocardiography to predict clinical complications: a meta-analysis. J Am Soc Echocardiogr 1997;10:562-8.

15. De Castro S, Magni G, Beni S, et al. Role of transthoracic and transesophageal echocardiography in predicting embolic events in patients with active infective endocarditis involving native cardiac valves. Am J Cardiol 1997;80:1030-4.

16. Carpenter JL. Perivalvular extension of infection in patients with infectious endocarditis. Rev Infect Dis 1991;13:127-38.
17. Anguera I, Miro JM, Vilacosta I, et al. Aorto-cavitary fistulous tract formation in infective endocarditis: clinical and echocardiographic features of 76 cases and risk factors for mortality. Eur Heart J 2005; 26:288-97.

18. Glazier JJ, Verwilghen J, Donaldson RM, et al. Treatment of complicated prosthetic aortic valve endocarditis with annular abscess formation by homograft aortic root replacement. J Am Coll Cardiol 1991; 17:1177-82.

19. McGiffin DC, Galbraith AJ, McLachlan GJ, et al. Aortic valve infection. Risk factors for death and recurrent endocarditis after aortic valve replacement. J Thorac Cardiovasc Surg 1992;104:511-20.

20. Sabik JF, Lytle BW, Blackstone EH, et al. Aortic root replacement with cryopreserved allograft for prosthetic valve endocarditis. Ann Thorac Surg 2002;74:650-9.

21. Avierinos JF, Thuny F, Chalvignac V, et al. Surgical treatment of active aortic endocarditis: homografts are not the cornerstone of outcome. Ann Thorac Surg 2007;84:1935-42.

22. Grover FL, Cohen DJ, Oprian C, et al. Determinants of the occurrence of and survival from prosthetic valve endocarditis. Experience of the Veterans Affairs Cooperative Study on Valvular Heart Disease. J Thorac Cardiovasc Surg 1994;108:207-14. 